\title{
Traduire l'ironie. L'exemple d'une ouvre romanesque de N. Kazantzaki et ses traductions française et anglaise
}

\author{
Maria Constantinou (Nicosie)
}

\begin{abstract}
This paper sets out to study the ironical effects related to morphological, rhetorical and macro-structural cues of the novel by N. Kazantzakis's $O \tau \varepsilon \lambda \varepsilon v \tau \alpha i o \varsigma ~ \pi \varepsilon \iota \varrho \alpha \sigma \mu o ́ \varsigma$ (1951), and then to examine the degree of their transposition in the French and English translations, carried out by Saunier (1959 La dernière tentation du Christ) and by Bien (1960 The Last Temptation of Christ) respectively.

Initially, from a theoretical point of view some definitions of irony as a thought and rhetorical figure are reviewed, while a particular attention is drawn to the theoretical insights of literary irony, in relation to Kazantzakis's work and life. From an analytical, enunciative perspective, this article will endeavour to locate, classify and analyze some techniques of ironization brought into play by Kazantzakis, with a view to comparing them with their transposition in the translated texts. The study privileges a pragmatico-textual approach, which embraces the theoretical background of enunciative polyphony.
\end{abstract}

\section{Cadre théorique}

\subsection{L'ironie comme phénomène énonciatif}

Lorsqu'on se propose d'étudier l'ironie, le premier problème qui surgit réside dans sa propre définition. En effet, la définition de l'ironie varie selon les auteurs ${ }^{1}$ et les traditions.

Selon une définition commune que l'on trouve chez Fontanier (1977: 145-146), l'ironie:

[...] consiste à dire par une raillerie, ou plaisante, ou sérieuse, le contraire de ce qu'on pense, ou de ce qu'on veut faire penser. Elle semblerait appartenir plus particulièrement à la gaieté; mais la colère et le mépris l'emploient aussi quelquefois, même avec avantage; par conséquent, elle peut entrer dans le style noble et dans les sujets les plus graves.

En ce sens, l'ironie est liée à l'antiphrase, et au déploiement de sentiments, et à des états d'âme. Or, au-delà de la définition classique de l'ironie qui consiste à provoquer une raillerie notamment par antiphrase, les chercheurs, (outre l'ironie situationnelle) associent l'ironie à la mention-écho, la contradiction argumentative, et à d'autres tropes (oxymore, métaphore etc.).

Cependant, les recherches les plus récentes mettent en exergue d'autres caractéristiques de l'ironie qui dépassent les limites du trope ponctuel ${ }^{2}$ et renvoient à un conflit de voix. Berrendonner (2002: 113) observe qu'il existe:

\footnotetext{
1 Parmi les travaux les plus importants, nous mentionnons: Kerbrat-Orecchioni 1976; Berrendonner 1982; Ducrot 1984; Schoentjes 1993; Perrin 1996; Landheer 1998 (Berrendonner 2002: 113).

2 D'ailleurs, toutes les ironies ne sont pas forcément d'antiphrases, ni de tropes, et par là même tous les tropes ne sont pas toujours ironiques.
} 
[...] beaucoup d'énoncés que l'on perçoit intuitivement comme ironiques, et qui ne se laissent aisément ramener à aucune des définitions existantes. Celles-ci ne recouvrent donc apparemment pas toute l'extension du phénomène tel qu'il est perçu par les récepteurs.

\subsubsection{Ironie littéraire, ethos et intentions de l'ironiste}

Dans cette optique, l'ironie est un phénomène énonciatif qui pose problème lors de son examen. C'est pourquoi il nous semble que pour comprendre si un énoncé est ironique, il faut tout d'abord cerner l'ethos et les intentions de l'auteur. Hamon (1996) rappelle que l'objectif de l'ironie ne consiste pas exclusivement à communiquer ou à partager une information: il consiste tout de même à partager "effectivement et réellement un auditoire ou un public de lecteurs" (1996: 125). En ce sens, l'ironie est "une communion qui se fait partiellement et sélectivement" au détriment d'un autre, une sorte d'examen de passage que "l'ironisant fait passer à son auditoire ou à ses lecteurs pour vérifier leur compétence idéologique." Il s'agit ici de l'ironie qui prédomine au théâtre, car l'ironisant se trouve devant son public. Mais elle n'est pas à exclure du roman, puisque le but du romancier est aussi de partager ses sentiments et communiquer avec son public.

En effet, l'examen de l'ironie suppose la prise en compte de la posture adoptée par l'ironiste même. Pour certains, l'ironiste est un hypocrite et un imposteur, tandis que pour d'autres c'est une personne sincère, désireuse d'abolir l'hypocrisie. Cela révèle un autre caractère de l'ironie: celui de la vertu, de la prudence et de la pudeur. Ainsi, Pierre Schoentjes ${ }^{3}$ (1993: 261) voit dans l'ironie une double sincérité:

Contrairement à la thèse trop souvent soutenue que l'ironie serait insincère, il semble donc que l'ironie soit doublement sincère: une première fois dans sa recherche d'un mode d'expression authentique, une seconde fois dans la distance qu'elle est capable d'instaurer entre subjectivité et l'expression artistique.

Jankélévitch de son côté, avance l'idée que l'ironie est un principe de prudence, de mesure et d'équilibre. En cela, elle n'est qu'un des visages de la pudeur,

cette précaution de l'âme qui ménage les transitions, espace les plans, empêche l'instinct de se jeter gloutonnement sur l'amour, en brûlant les étapes (Jankélévitch 1997: 166).

Kazantzaki oscille entre les deux, nous semble-t-il. Il est ironiste par excellence envers tout dogme, toute doctrine voulant cantonner le divin et l'humain dans une étroitesse d'esprit, dans l'hypocrisie, le mensonge et la méchanceté. D'une part, on voit chez Kazantzaki le refus de la demi-mesure, de la "médiocrité de l'âme", de la mesquinerie, du snobisme, et de l'arrogance, ce qui lui confère la figure d'un apostat, d'un dissident. D'autre part, on observe chez lui une personne profondément croyante, sincère et humaine. Et tout cela se déploie dans toute l'œuvre. Il y a d'un côté, la patience exprimée notamment à travers le visage de Jésus, et de l'autre, l'inquiétante étrangeté, la précarité menaçante qui renforce le sentiment de colère et de révolte. En effet, Kazantzaki "fait exploser" ses personnages; le centurion, Judas, Madeleine, la vieille dame, et bien d'autres.

Sous cet angle, l'ironie est à saisir comme une sorte d'éthique qui interroge les éléments divin et humain. D'ailleurs, l'ironie sollicite du lecteur un rôle actif. Elle implique l'interrogation,

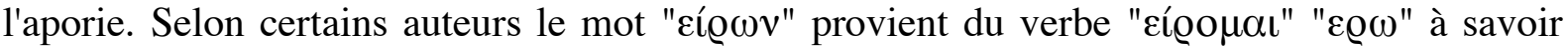
"interroger". Partant de cette "version étymologique", Preminger avance l'idée qu'"il faut que le lecteur s'interroge sur ce qu'on a pu vouloir... Telle est l'ironie au sens strict." (cité par Dupriez 1984: 264). Elle porte à la fois sur la louange et le blâme; deux formes de l'épidictique qui s'interposent et s'opposent à la fois dans tout l'univers romanesque.

\footnotetext{
${ }^{3}$ L'auteur avance l'idée que "l'ironie est à la fois création de subjectivité originale et distanciation critique par rapport à cette subjectivité" (ibid.)
} 


\subsubsection{Polyphonie énonciative; l'ironie à l'œuvre kazantzakienne}

Kazantzaki expérimente une ironie qui se mêle à l'humour, mais aussi à l'amertume, à la colère, à la "profanation" et à la révolte face à l'iniquité humaine et "divine". Le romancier grec porte sur le monde un regard à la fois ludique et critique. Chaque personnage incarne différents rôles et exprime des sentiments divers. Cela suppose la prise en compte de "qui parle" et "comment il parle", ainsi que "qui pense".

La perspective énonciative de l'ironie romanesque implique par là même la notion de polyphonie. La polyphonie concerne les rapports multiples qu'entretiennent l'auteur, le narrateur, les personnages, les voix anonymes (le "on-dit"), les différents niveaux stylistiques qui se superposent et s'opposent en même temps (Bakhtine 1978; Ducrot 1984). Ainsi, la marque de chacun des personnages du roman kazantzakien s'affirme-t-elle à travers la matérialité signifiante, c'est-à-dire au niveau des registres de langue, des figures discursives, des jeux de mots, bref, tout ce qui donne corps au sens et à la signifiance du texte.

\subsubsection{Indices, réseaux textuels et typologie des effets ironiques}

L'ironie, en tant que figure macro et micro-structurale, contribue à la construction des réseaux textuels. Elle consiste à la fois en un mode d'énonciation global et local. Global, car tout le texte est ironique et local, car le narrateur, les personnages produisent un effet d'ironie dans un endroit précis du texte (Hamon 1996: 82).

Les signaux de l'ironie pour Hamon peuvent "se distribuer, a priori, à tous les niveaux (morphologique, typographique, rhétorique, syntaxique, rythmique, lexical) et à tous les endroits du texte." (Hamon ibid.: 80).

Pour accentuer l'ironie, Kazantzaki a recours à des phénomènes morpho-sémantiques (diminutifs et augmentatifs), à des figures discursives (dérivation, synecdoque, métaphore etc.) et à des expressions idiomatiques à contenu injurieux etc.

Cependant, l'ironie ne s'actualise qu'en fonction du contexte, et dans l'espace polyphonique dans lequel elle s'inscrit. Ce qui exige un mouvement de va-et-vient entre "voix" et "langue".

Compte tenu de l'espace dont nous disposons ici nous nous limiterons à quelques indices formels d'ironisation qui présentent un certain intérêt traductologique, tout en prenant en considération la polyphonie énonciative dans laquelle s'actualise l'ironie. Nous choisissons d'étudier quelques diminutifs et augmentatifs, le phénomène de dérivation vue ici comme figure discursive et stratégie très fréquente chez N. Kazantzaki et la métaphore.

Il est évident que plusieurs phénomènes sont mis de côté, tels que le contraste des niveaux de langue, les jeux de mots etc. Pour cette raison, il est juste de dire que le présent travail est moins comme une tentative de retracer un parcours que comme l'amorce pour une approche textuelle de l'ironie dans une perspective traductologique.

\subsection{Le traducteur face à l'ironie}

Du point de vue traductologique on se demande comment se traduisent ou se transposent les effets ironiques d'une langue à l'autre. Faut-il toujours respecter la matérialité signifiante (diminutifs, dérivation, images, métaphores) pour reproduire l'ironie dans le texte traduit? Estce qu'une divergence au niveau discursif, engendre-t-elle toujours des entropies à l'égard des effets ironiques? D'autre part, est-il question de langue ou de discours, et par là même quelle est la part de la subjectivité du traducteur?

L'ironie comme figure discursive, n'est pas de l'ordre de la langue mais de l'ordre du discours. Elle est donc affaire subjective impliquant par là même un sujet-énonciateur (l'écrivain) qui la 
produit et un sujet-énonciataire (le lecteur) qui la reçoit. Et c'est en effet, entre ces deux instances, l'une émettrice et l'autre réceptrice que le traducteur oscille. D'abord, étant lecteurrécepteur et ensuite ré-écrivain -émetteur, le traducteur est appelé à comprendre, interpréter et reproduire les effets ironiques. Mais par où commencer, et à quels critères doit-il répondre pour effectuer une bonne traduction? Quelles stratégies de traduction impliquerait le phénomène d'ironie?

Puisque le roman est avant tout un texte littéraire, il faut le lire comme un texte littéraire. Cela suppose un regard attentif à tout élément, car tout y fait signe. L'écoute à la lettre ${ }^{4}$ s'avère parfois imposée. Le traducteur doit donc partir de l'écrivain même, de ses "empreintes d'écriture", son style. En l'occurrence, Kazantzaki invente des mots, des figures discursives, etc. pour créer de l'humour, ironiser et faire réfléchir. A cette inventivité réfléchie de l'écrivain grec, le traducteur est censé répondre par une autre inventivité réfléchie, afin de créer chez le public visé les mêmes ou presque les mêmes effets ironiques. Pour ne pas annuler le "contrat draconien", le traducteur se doit de créer voire recréer sur les traces de l'auteur. De la sorte, il est appelé à respecter la matérialité signifiante, la créativité lexicale, le style de l'auteur, l'implicite, les connotations.

Pour voir de plus près et "sur le tas" les problèmes que pose la traduction de l'ironie, nous proposons une analyse de quelques exemples. L'analyse se construit de la façon suivante: a) indices morphologiques, b) images/métaphores et c) dérivation.

\section{$2 \quad$ Analyse des exemples}

\subsection{Indices morphologiques: diminutifs et augmentatifs comme signaux de dérision}

Dans l'œuvre de N. Kazantzaki certains diminutifs sont susceptibles d'enclencher de l'ironie. Ainsi, Zébédée, s'adressant à ses pêcheurs qui travaillent pour lui, prononce les mots suivants:

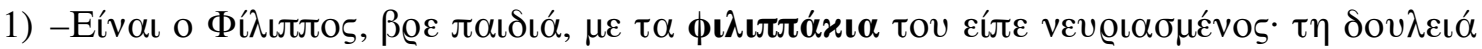

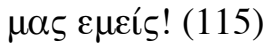

-C'est Philippe, les enfants, avec sa philippaille! dit-il, énervé. Nous autres, occupons-nous de nos affaires! (113)

"It's Philip and his philipkins", he said with irritation. "As for us, back to work!" (107)

L'écrivain, à partir du nom propre $\Phi i \lambda \iota \pi \tau o \varsigma$, forge à l'aide du suffixe - $\alpha \varkappa \iota$, le mot $\phi \iota \lambda \iota \pi \tau \alpha \dot{x} \iota \alpha$. Le suffixe, pris dans le système linguistique, serait indiscutablement lié à

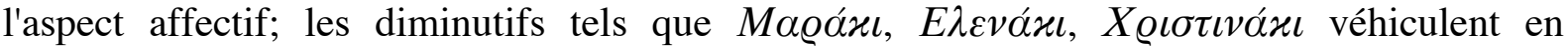
fonction du contexte des connotations émotionnelles. Or, il convient de noter que Zébédée, l'un des personnages du roman, qui incarne l'hypocrisie, l'avidité, la ruse et l'individualisme, exprime un certain mépris envers autrui. En effet, ce qui l'intéresse ce sont ses affaires. Ce qui peut aller à l'encontre de son propre intérêt l'irrite et l'agace. Philippe lorsqu'il voit les pêcheurs, siffle pour leur faire signe, mais Zébédée le décourage: "Nous avons du boulot, Philippe, soit gentil, va-t-en" en lui tournant le dos.

L'enjeu dans la traduction réside dans la restitution de ce mépris exprimé par le diminutif grec, qui situé dans le contexte précis engendre des connotations ironiques. La traduction de Saunier est jugée aussi intelligente que créative. Dans le sillage de l'écrivain, le traducteur se décide à rendre la traduction, étrange, "excentrique", afin de faire revivre les mêmes ou presque les mêmes effets ironiques. On l'a vu, le mot grec est une invention de l'écrivain pour marquer l'indifférence, le mépris des personnes, telles que Zébédée envers autrui. L'ironie se

\footnotetext{
${ }^{4}$ Et cela n'implique nullement la réduction de la traduction de la lettre au mot à mot. D'ailleurs, le mot à mot à proprement parler est très souvent inacceptable. A ce sujet voir Berman (1985: 14-15).
} 
déploie à deux niveaux: celui du personnage qui parle, c'est-à-dire Zébédée envers les autres et celui de Kazantzaki envers des personnes qui ressemblent à Zébédée. La traduction est inventive et semble accomplir ce que Meschonnic (1999: 460)5 appelle "invention de discours" qui est "le rapport très fort et caché entre écrire et traduire."

Cette inventivité n'est pas à isoler de la langue. A y regarder de plus près, le traducteur français, devant la créativité de Kazantzaki, cherchera des outils linguistiques pour rendre des connotations analogues; le recours créatif au système linguistique donnera naissance à philipaille. Le traducteur ne crée pas dans le vide, mais crée avec et contre sa langue. L'unité linguistique -aille inhérente à la langue française, est négativement marquée dans l'inconscient du locuteur francophone car elle renvoie à racaille, canaille, mangeaille etc. Quant au traducteur anglais, comme son homologue français, il met en œuvre la créativité lexicale que son système linguistique lui permet pour innover à la manière de l'écrivain grec. De la sorte, il jonglera avec l'élément -kin qui se comporte comme un élément autonome en langue anglaise et qui veut dire "parents", "famille", "proches". Il peut comporter donc des connotations péjoratives.

Bien que l'ensemble de l'œuvre de Kazantzaki se distingue par son humanité, il est à noter que cet amour touche également à l'impuissance et à la faiblesse humaine. L'entrelacement de mots ayant la même racine en est un exemple privilégié. Judas chez Kazantzaki se met à rire et prononce les mots suivants:

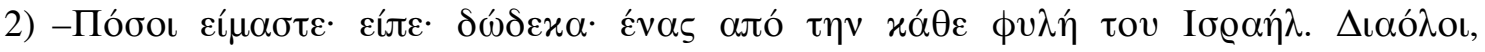

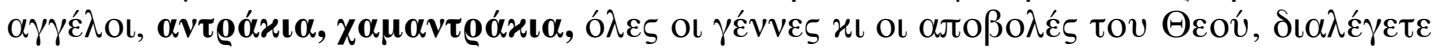

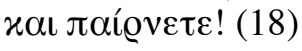

-Combien sommes-nous? Dit-il. Douze; un de chaque tribu d'Israël. Diables, anges, nains, nabots, tous les enfants et tous les avortons de Dieu, choisissez et emportez? (16)

"How many are we?" he asked. "Twelve - one from each of the tribes of Israel. Devils, angels, imps, dwarfs: all the births and abortions of God. Take your pick." (10)

Le personnage en promenant son regard sur la troupe disparate dit que c'est bien Jésus "l'aiguillon", alors qu'eux (nous les autres) sont "les bœufs de labour". La présence des diminutifs marque cette impuissance. Les substantifs $\alpha \nu \tau \varrho \alpha ́ \varkappa \iota \alpha, \chi \alpha \mu \alpha \nu \tau \varrho \alpha ́ \varkappa \iota \alpha$, suffixés en - $\alpha \varkappa \iota$, qui riment d'ailleurs, créent un autre réseau lié à la faiblesse humaine. Le lecteur grec est appelé à jouer un "rôle interrogatif", afin d'imaginer et d'actualiser des connotations qui se trouvent sous-jacentes dans la matérialité du signifiant. Le signifiant $\chi \alpha \mu \alpha v \tau \varrho \alpha ́ x \iota \alpha$, en enclenchant une exploration des ressources linguistiques invite le lecteur à l'"enquête du sens". Son étymon est susceptible d'engendrer d'autres réseaux liés à l'ironie. Le mot provient

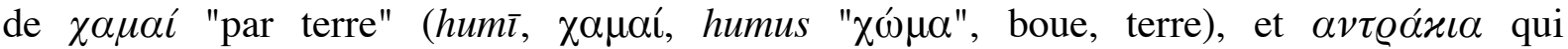
littéralement signifierait "petits hommes" mais aussi des hommes (des gens) sans pouvoir, sans autorité, sans puissance, naïfs, ingénus, innocents. Du point de vue de la réception, le lecteur grec peut percevoir - outre la gradation sémantique qui s'exerce sur l'axe syntagmatique dans $\alpha v \tau \varrho \alpha ́ x \iota \alpha$ et $\chi \alpha \mu \alpha \nu \tau \varrho \alpha ́ x \iota \alpha^{6}$ - une certaine ironie qui ne se transmet que partiellement dans le texte traduit. En observant de plus près le texte grec, on voit que $\alpha \nu \tau \varrho \alpha ́ x \iota \alpha$, et $\chi \alpha \mu \alpha \nu \tau \varrho \alpha ́ x \iota \alpha$ proviennent du mot $\alpha ́ v \tau \varrho \alpha \zeta$, signifiant "homme". Il s'agit d'un élément que l'on rencontre de façon récurrente dans l'œuvre de Kazantzaki et qui reflète le monde "phallocratique" de l'écrivain.

\footnotetext{
${ }^{5}$ Meschonnic soutient que la traduction doit: "être une invention de discours, si ce qu'elle traduit l'a été" (1999: 460).

${ }^{6}$ L'agencement de ces deux mots favorise une gradation sémantique, vu que le deuxième est sémantiquement plus fort que le premier.
} 
Compte tenu de l'univers de l'écrivain, les mots en question connotent que l'être humain est faible et fragile et que cette fragilité humaine conduit à l'avilissement et la décadence de l'homme devant l'existence du divin. Le lecteur retrouve la même gradation à plusieurs reprises, dans des contextes qui renforcent notre hypothèse herméneutique:

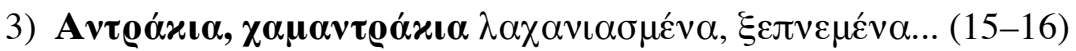

Des nains, des nabots, haletants, à bout de souffle; (13)

They were panting dwarfs, imps gasping for breath... (8)

Le narrateur ici se réfère au jeune homme, le Christ qui dans son sommeil voit "les terres saintes, les eaux sacrées" et tend la main pour les toucher. Or le rêve s'estompe lorsque la Terre Promise, "faite de fraîcheur et de vent et d'antique désir humain", se met à trembler, et s'éteint. Et à ce moment le jeune homme entend des voix rugissantes, voit "la meute aux mille têtes", les colosses flétris et ratatinés, ces "nains", et "nabots".

A l'échelle de l'œuvre, ces énoncés s'associeraient, en établissant des isotopies ${ }^{7}$, au motobsession $\lambda \alpha \dot{\sigma} \sigma \eta \eta$ "boue". "Boue" dans l'espace romanesque engendre des connotations qui renvoient à l'état primitif mais aussi au destin inéluctable auquel aucun être humain ne peut échapper (Constantinou 2006: 249, 276 seqq.).

Dans le texte traduit la gradation sémantique de nains et de nabots, avec les groupes adjectival et prépositionnel connote le néant, le manque de puissance, l'inanité humaine. Par ailleurs, un rapprochement phonétique pourrait se faire entre nains, nabots et néant. Il y a donc densification sémantique qui traduit ou exprime à son tour une ironisation. La traduction anglaise qui recourt aux mots imps et $d w a r f s$ ne reproduit pas les mêmes connotations que la traduction française bien qu'elle semble se rapprocher plus de celle-ci que de l'original.

Les augmentatifs qui connaissent un taux de fréquence très élevé dans l'œuvre romanesque sont des indices d'ironie.

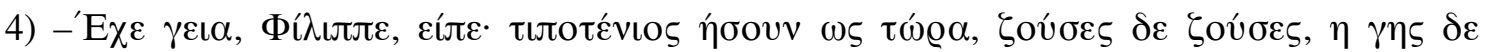

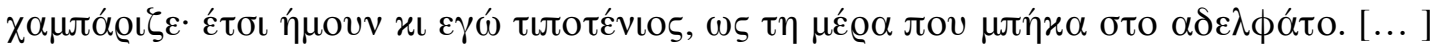

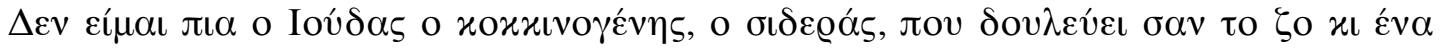

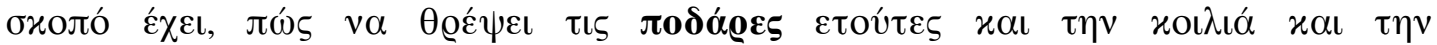

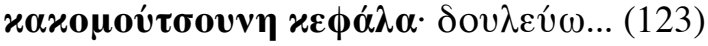

-Adieu, Philippe, dit-il, jusqu'à présent tu n'étais rien du tout, le monde ne savait pas si tu vivais ou non. J'étais comme ça moi aussi, un rien du tout, jusqu'au jour où je suis entré dans la confrérie. [...] Je ne suis plus Judas le rouquin, le forgeron, qui travaille comme un bête de somme et qui n'a qu'une idée: comment nourrir ces grands pieds, ce ventre et cette sale gueule. $(120-121)$

"Farewell, Philip. You were a mere nothing until now; no one cared whether you were dead or alive. I was the same - a nothing - until the day I entered the brotherhood, [...]. No more Judas the redbeard, the blacksmith who slaved like an ox with the sole purpose of nourishing these feet and this belly and this ugly snout." (115-116)

Judas, disant adieu à son frère Philippe, lui confesse qu'avant d'entrer dans la confrérie, il était un rien du tout. Son seul souci était comment se nourrir, nourrir ses $\pi \circ \delta \alpha$ $\varrho \varepsilon \varsigma$ ("grands pieds"), sa $\varkappa \varepsilon \phi \alpha ́ \lambda \alpha$ ("sale gueule")! Etant donné que la langue française ne dispose pas d'augmentatifs pour ces deux substantifs, le traducteur recourt au syntagme grands pieds et à

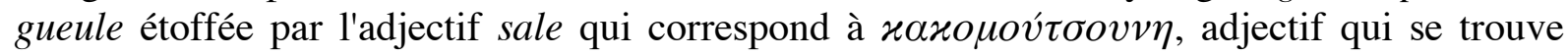

\footnotetext{
${ }^{7}$ En effet, l'isotopie est la récurrence d'un même sème appelé isotopant qui s'actualise dans différents sémèmes au sein d'un syntagme, d'une phrase ou d'un texte. La réitération de ces suites d'éléments linguistiques, construit l'homogénéité discursive (Rastier 1987, 1989; Jeandillou 1997).
} 
dans le texte grec et qui détermine l'augmentatif $\varkappa \varepsilon \phi \alpha ́ \lambda \alpha$ "grosse tête". Pour intensifier les effets ironiques le traducteur recourt également à l'embrayeur "cette". La langue anglaise oblige aussi le traducteur à faire appel à d'autres solutions. Ainsi, les embrayeurs "these" et "this" qui actualisent ici le sème /intensité/ contribuent à l'ironisation.

Dans l'espace textuel, l'ironie se situe sur un double plan: d'une part, Judas qui exerce une "auto-dérision" et, d'autre part, celui qui pense, à savoir l'auteur qui vise ceux n'ayant pas de cause dans leur vie et dont le seul souci est comment "se nourrir" au quotidien.

Les exemples ci-dessus mettent en évidence l'importance des indices formels de l'ironie. Les traducteurs répondent avec brio aux exigences du texte à ré-exprimer. L'infléchissement de la lettre, le jonglage avec les mots, le recours à des éléments qui n'existent pas dans le texte original (déictiques par exemple) semblent transférer l'ironie du roman de Kazantzaki.

\subsection{Images et métaphores comme processus d'ironisation}

Comme nous l'avons dit, l'œuvre de Kazantzaki foisonne en images et métaphores qui, sous forme d'adjectifs ou d'expressions tendent à intensifier les effets ironiques.

Ainsi, pour caractériser Simon d'ivrogne, Pierre produit l'énoncé suivant:

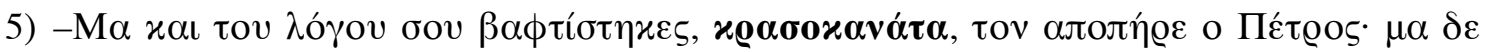

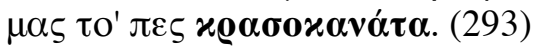

-Mais toi aussi tu t'es fait baptiser, sac à vin, lui dit vertement Pierre, tu ne nous l'as pas dit? (290)

"But you were baptized too, wine jug!" Peter scolded him. "You told us yourself. [...]" (283)

Il s'agit du dialogue entre Pierre et Simon où le dernier s'insurge contre Jean Baptiste qu'il qualifie de faux prophète. Pierre le reprend en lui rappelant que lui aussi (Simon) a été baptisé en cachette. Le recours à l'image $\varrho \alpha \sigma o \varkappa \alpha \nu \alpha ́ \tau \alpha \varsigma$ qui correspond parfaitement à sac à vin, et wine jug renforce les réseaux textuels liés à l'ironie. Sac à vin comme son homologue anglais et grec mettent en place le processus synecdochique lié à l'ivresse. Le "référent" intervient comme image favorisant un transfert sémantique qui dynamise le sens du texte. Les trois langues disposent du même mot-image pour exprimer la même réalité et créer des effets d'ironie.

Dans le roman en question, le mépris vise souvent Madeleine. Cependant, notons qu'il ne s'agit pas d'un mépris partagé par l'auteur, mais par les autres personnages, auxquels l'auteur semble s'opposer. A travers une image, chargée de contenu sexuel, Nathanaël dit:

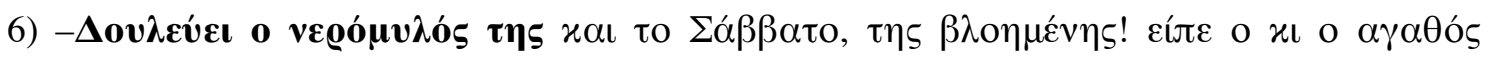

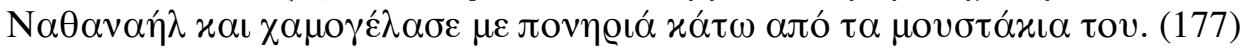

-Son moulin travaille même le samedi, à la mâtine! fit le candide Nathanaël et il sourit malicieusement sous sa moustache. (175)

"Her water mill is open on the Sabbath too, bless her!" said simple Nathanael, smirking craftily in his beard. (170)

Les mots s'adressent à Philippe, qui lui premier dit à Nathanaël avec ironie: "Qu'elle soit la bienvenue parmi nous, les enfants!" Avec un sourire malicieux, Nathanaël emploie une image pour connoter qu'elle "travaille" même le samedi! Son moulin travaille est une traduction littérale qui rend parfaitement l'ironie de l'oeuvre originale. La traduction anglaise, en dépit du déplacement qu'elle opère (her water mill is open), rend également avec réussite les effets

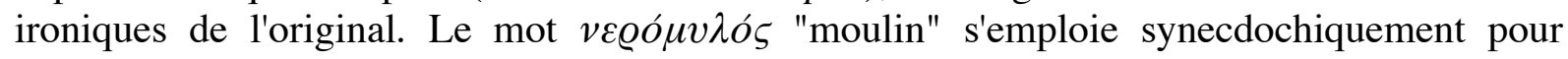
signifier le sexe de Madeleine. La fidélité au même référent facilite la transposition des effets ironiques dans les autres textes. 
Les moments ironiques ne sont pas détachés les uns des autres. Ils s'enchaînent et se construisent en réseaux quelques pages plus loin:

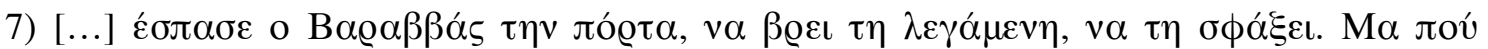

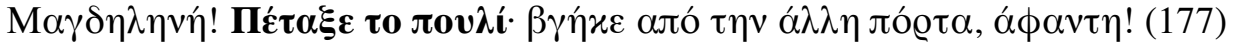

Barabbas a brisé la porte pour trouver la belle en question et l'égorger. Seulement, plus de Madeleine! L'oiseau s'était envolé. Elle était sortie par l'autre porte, ni vu ni connu! (174175)

Barabbas broke down the door to find the lady in question and slaughter her. But where was Magdalene? She'd flown the coop, gone out through the back door, unseen! (170)

Le narrateur décrit la scène où Barabbas cherche Madeleine pour l'égorger. L'expression

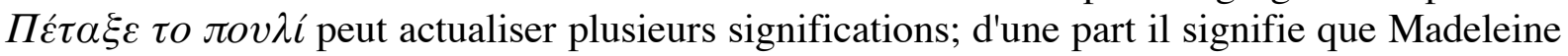
a disparu, d'autre part que Barabbas a raté la chance de l'égorger. L'expression française l'oiseau s'était envolé s'emploie pour dire que celui qu'on recherchait, s'est enfui. Le texte anglais opte pour to fly the coop "s'envoler", qui s'utilise pour désigner quelqu'un qui a quitté un lieu où il se sentait "emprisonné". D'ailleurs coop correspond à poulailler, cage, qui synecdochiquement renvoie à prison. Les trois textes ont donc ceci de commun: ils actualisent le sème /animal/ (oiseau/poulailler) qui n'est pas sans véhiculer des connotations péjoratives.

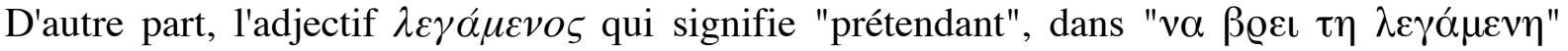
contribue à l'ironisation. L'ironie se transpose par le biais des syntagmes nominaux "la belle" et "the lady in question" respectivement.

Ce processus métaphorique lié à l'intensification de l'ironie se déroule dans l'ensemble du roman. Ainsi, les paroles du Judas de Kazantzaki deviennent plus ironiques:

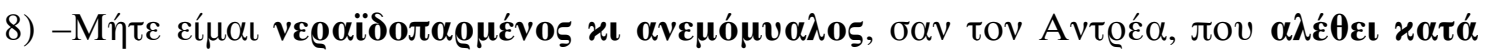

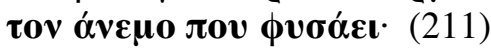

-Je ne suis pas non plus un visionnaire ni une cervelle éventée, comme girouette qui tourne avec le vent. (207)

"I'm not a scatter-brained daydreamer like Andrew, who changes his mind with every breeze that blows. [...]" (203)

Judas, pour montrer à Jésus qu'il n'a rien à voir avec "ses gens", ses disciples, se compare avec

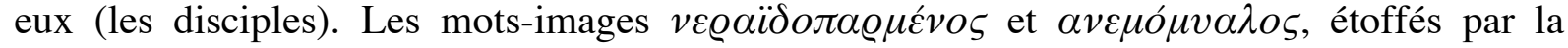

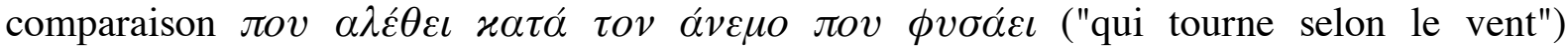
concourent à l'ironisation. Judas en effet, veut se démarquer d'eux. Les traductions semblent transposer l'ironie grâce aux métaphores et images. D'une part, "une cervelle éventée, comme girouette qui tourne avec le vent", et d'autre part, le texte anglais avec "scatter-brained daydreamer [...] who changes his mind with every breeze that blows."

L'étude comparative de ces quelques exemples nous amène à déduire que la fidélité à la lettre, au référent et aux connotations concourt à la transposition de l'ironie. Les images et métaphores demandent à être reproduites dans les traductions pour que les effets ironiques se réactualisent sur les autres rives.

\subsection{Dérivation et intensification}

La dérivation se met au service de l'ironie, pour marteler ici l'idée d'exagération et de contraste. En grammaire traditionnelle, la dérivation est "un procédé de formation de mots nouveaux par modification (addition, suppression, ou remplacement) d'un morphème (suffixe) par rapport à une base" (Le Petit Robert). Cependant, en rhétorique, le terme de dérivation s'emploie pour désigner ce processus qui consiste "à utiliser dans une même phrase des mots, 
L'exemple d'une œuvre romanesque de N. Kazantzaki et ses traductions française et anglaise

dérivés d'un même radical" (Ricalens-Pourchot 2003: 154). Autrement dit, elle vise à rapprocher dans le discours des mots appartenant à la même famille (Bacry 1992: 196).

Comme nous allons le voir, il ne s'agit point de pénurie lexicale, mais d'un emploi délibéré, dans le but de créer des effets de sens, et de marteler une idée. La dérivation est ici liée à l'intensification, et notamment à l'accentuation des effets ironiques. Ainsi, le fils de Marie se demande:

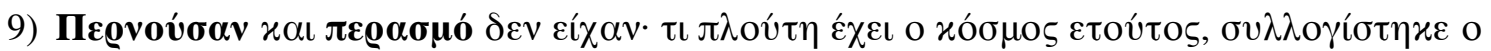

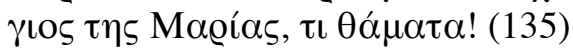

Ils passaient, passaient sans fin. Que de richesses en ce monde, pensa le fils de Marie, que de merveilles! (132)

The procession filed by; it seemed to have no end. What riches these people have, the son of Mary thought, what marvels! (127)

Le narrateur ironise à travers la pensée du fils de Marie le paradoxe du monde. On voit d'un côté, les riches avec leurs marchandises précieuses, de l'autre, l'humble petit âne et les esclaves. La dérivation renforce ce paradoxe. En outre, la phrase exclamative "Que de richesses en ce monde [...] que de merveilles!" peut se considérer comme une anti-phrase qui marque l'iniquité et l'injustice. La dérivation renvoie également plus bas aux riches marchands qui vont s'arrêter à Magdala. Ce qui fait frissonner le fils de Marie. (-Ils vont s'arrêter à Magdala, pensa-t-il subitement, ils vont s'arrêter à Magdala). Alors que le texte grec met en oeuvre une dérivation à base d'un verbe et d'un substantif, ayant la même racine, la traduction française recourt à un doublement du verbe "passaient, passaient" avec le syntagme prépositionnel "sans fin". Certes, une traduction mot à mot s'avère ici impossible, compte tenu des divergences entre les langues. Le doublement du verbe permet de créer d'autres effets de sens liés à l'ironie. En revanche, la traduction anglaise n'accentue pas l'ironie. "The procession filed by; it seemed to have no end," explicite le texte mais ne rend pas les mêmes connotations. Il s'agit d'une explicitation. Or, comme le remarque Arsaye (2004: 384): "expliciter ce que la langue-source exprime de manière implicite peut détruire l'ironie."

La dérivation comme stratégie très fréquente chez l'écrivain grec sert à accentuer les connotations ironiques qui se construisent en réseaux. Ainsi, un vieil homme s'adresse à Jésus et l'invite à l'accompagner à "Magdala" (chez Madeleine). Ses propos sont secs, burlesques et mordants:

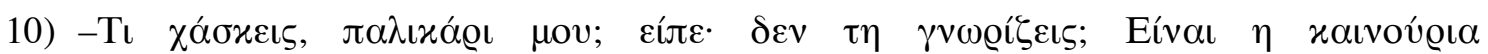

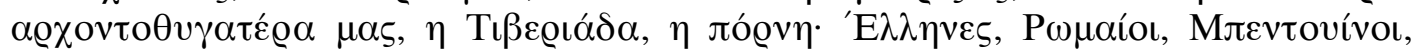

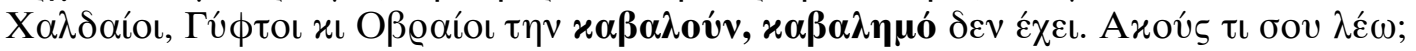

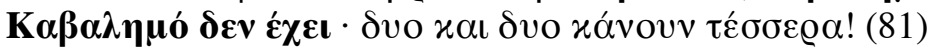

-Pourquoi restes-tu là bouche bée, mon garçon? dit-il, tu ne la connais pas? C'est notre nouvelle princesse, Tibériade la putain; les Grecs, les Romains, les Bédouins, les Chaldéens, les Bohémiens, les Hébreux lui montent dessus, ça ne la mate pas. Tu peux crois ce que je te dis, ça ne la mate pas; deux et deux font quatre! (79-80)

"What are you gaping at, lad?" he asked. "Don't you know her? She's our new princess: Tiberias the whore. Greeks, Romans, Bedouins, Chaldeans, Gypsies and Jews mount her, and she's always ready for more. She's always ready for more - do you hear what I say? Two and two make four!" (75)

La dérivation sert à intensifier l'ironie et à provoquer le rire. Une traduction littérale s'avère impossible, vu que les deux langues, française et anglaise ne disposent pas de la même flexibilité que le grec. Cependant, l'ironie blessante se transpose de façon adéquate grâce à la répétition des mots et des phrases qui fonctionnent ici comme figures discursives et servent à accentuer les effets ironiques. 
Cependant, dans d'autres exemples, les effets ironiques semblent affaiblis lors du truchement.

Le narrateur se réfère aux disciples. Ils paraissent inertes, sans volonté. Ils semblent être entièrement soumis à Jésus:

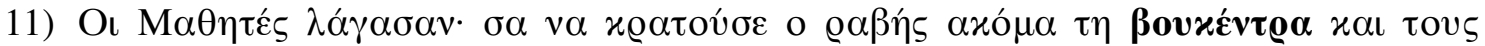

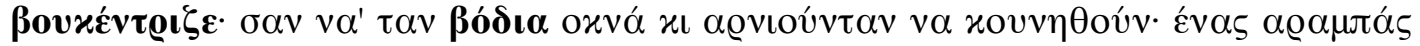

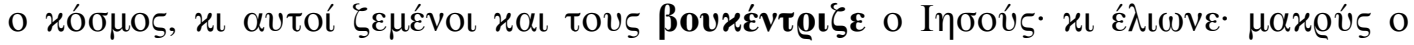

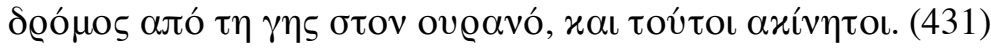

Les Disciples se tinrent cois. Il semblait que le maître tenait encore l'aiguillon et les en frappait, et qu'ils étaient, eux des bœufs indolents qui refusaient de remuer. Le monde est un chariot, ils y étaient attelés, Jésus les aiguillonnait, et ils renâclaient, ils ne bougeaient pas. Jésus les regardait et s'épuisait. La route est longue de la terre au ciel et ils restaient immobiles. (427-428)

The disciples cowered. It was as thought the rabbi still held the ox-goad and pricked them, as though they were sluggish oxen who refused to move. The world was a cart to which they were yoked. Jesus goaded them on, and they shifted under the yoke but did not budge. Looking at them, Jesus felt drained of all his strength. The road from earth to heaven was a long one, and there they were, motionless. (415)

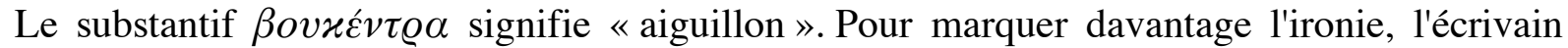

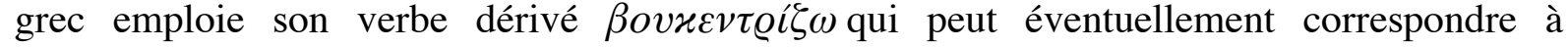
«aiguillonner». Le lecteur hellénophone se trouve devant un jeu discursif fondé sur le procédé de dérivation qui donne lieu à un pléonasme. Notons que le mot n'est pas répertorié dans le dictionnaire, ce qui porte à penser que le verbe n'existe pas en langue (système fonctionnel) et qu'il est soit un mot dialectal, soit un mot forgé par l'écrivain même. Quoi qu'il en soit, cette stratégie discursive vise à inviter le lecteur à un rôle interrogatif. La prise en compte du tempérament de l'écrivain qui se veut toujours "en action" nous amène à conclure qu'il s'agit d'une dérivation ayant pour finalité d'ironiser les personnes sans volonté ${ }^{8}$, qui n'agissent pas ou ne réagissent pas.

Les traductions ne véhiculent pas les mêmes effets ironiques. Quant à la traduction française, on remarque une certaine atténuation, puisque ce jeu de mots ne se reproduit pas. En effet, la langue française dispose des possibilités aussi spectaculaires que la langue de Kazantzaki, vu que aiguillonner est le dérivé du substantif aiguillon. On se demande pourquoi ne pas procéder à une dérivation "le maître tenait encore l'aiguillon et les aiguillonnait". Le texte anglais contient plusieurs indices d'ironie. Le traducteur, grâce à ses choix stylistiques, réussit à produire un texte riche en connotations ironiques. Précisément, ox-goad fait écho au verbe to goad et ox- à oxen ("bœuf"). D'autre part, yoke qui est à la fois un substantif et un verbe, enrichit davantage le mécanisme d'ironisation. Le traducteur anglophone se focalise sur l'emploi répétitif de ces quelques éléments pour densifier l'ironie.

L'ironie devient plus forte et plus "profanatrice" lorsqu'elle touche à l'élément divin. Marie et le rabbin cherchent à savoir où se trouve Jésus. Marie inquiète et désespérée, poussant un cri, prononce un blasphème:

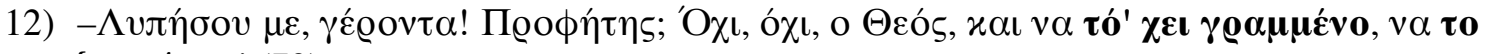

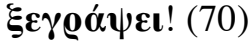

-Aie pitié de moi, vieillard! Un prophète? Non, non, que Dieu, s'il l'a écrit, l'efface! (69)

"Have pity on me Father! A prophet? No, no! And if God has it so written, let him rub it out! [...]" (64)

\footnotetext{
8 Il est à noter que l'ironie ne cible pas Jésus.
} 
Elle souhaite que son fils soit comme les autres êtres humains, non un prophète. Du point de vue linguistique et stylistique, on remarque que la dérivation résulte de la co-présence des deux antonymes: $\gamma \varrho \alpha ́ \phi \omega$ et $\xi \varepsilon \gamma \varrho \alpha ́ \phi \omega$. Il s'agit d'un mécanisme qui nous rappelle d'autres cas en français ("apprendre/désapprendre", "tromper/détromper" etc.). L'opposition s'exerce dans le préfixe $-\xi \varepsilon$ - élément qui indique la privation, l'éloignement, la séparation. Le verbe $\gamma \varrho \alpha ́ \phi \omega$

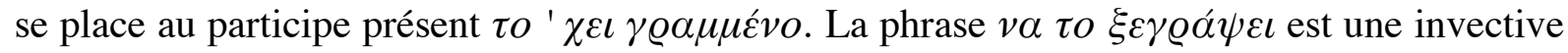
qui contribue à la densification des réseaux. En effet, il s'agit bel et bien de profanation susceptible de heurter le sentiment religieux du lecteur hellénophone, notamment chrétien orthodoxe.

Dans les traductions l'ironie liée à la profanation n'est pas passée sous silence; les phrases "l'efface" et "let him rub it out" sont des invectives qui reproduisent les effets ironiques. Cependant, compte tenu du jeu de dérivation, on dirait que l'ironie est plus frappante dans l'original.

Par ailleurs, le même mécanisme se produit dans la même page, quelques lignes plus haut. Le lecteur est devant les paroles du vieux rabbin avec qui Marie dialogue. Etourdi, éperdu, dans ses lectures de la Bible, il avoue que sa sagesse ne sert plus à rien. Dans le désarroi, il dit qu'il "plie" et "déplie" ("enroule" et "déroule") les Ecritures:

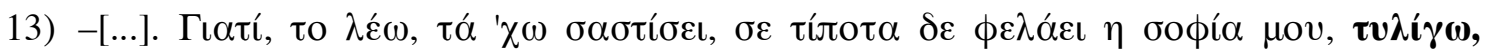

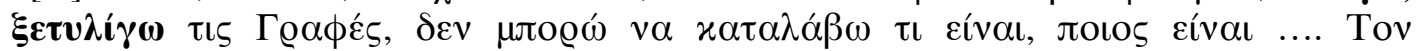

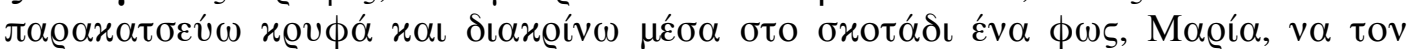

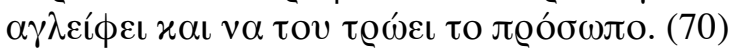

-[...]. Parce que, je te le dis, j'en ai le vertige, ma sagesse ne sert à rien, j'ouvre et je ferme les Ecritures, je ne peux pas comprendre ce qu'il est, qui il est...je le guette en cachette et je distingue dans l'obscurité une lumière, Marie qui lui lèche, qui lui dévore le visage. (68)

"[...] - I am completely confused; my knowledge is of no help whatsoever: I unroll the Scriptures tirelessly but I cannot comprehend what or who he is. I spy on him in secret, therefore, and in the darkness I discern this light which licks him and devours his face. [...]"

Les deux traductions s'avèrent personnelles et interprétatives. D'un côté, le traducteur francophone rend ce processus de dérivation par le couple antonymique ouvrir/fermer, de l'autre, le traducteur anglo-saxon préfère garder le verbe unroll ("dérouler") en rendant son opposé par l'adverbe tirelessly ("infatigablement").

On observe une atténuation du point de vue de la figure employée délibérément par l'écrivain et une densification grâce à la présence de l'adverbe en question qui semble étoffer le verbe "unroll". Or, on se demande pourquoi ne pas reproduire "I roll and unroll" ou "j'enroule et déroule les Ecritures", pour provoquer les mêmes effets qui attirent peut-être plus le regard du lecteur. Car, comme on l'a vu, l'explicitation risque d'inhiber les traits ironiques.

D'ailleurs, à y regarder de plus près, on peut constater une tentative d'embellissement de la structure signifiante, en utilisant des signifiants qui s'éloignent phonétiquement, par un souci de mieux écrire. Et c'est peut-être ce que Chevalier appelle "sentiment de l'orthonymie" (1995: 60), principe de la plupart de traducteurs qui préfèrent recourir à des formulations habituelles, correctes pour ne pas heurter le "génie de la langue".

\section{$3 \quad$ En guise de conclusion}

De cette comparaison de langues et de textes on peut déduire que l'ironie, dans une perspective traductologique ne peut être envisagée que du point de vue énonciatif, tout en prenant en compte la microstructure du texte. Ce qui nécessite la prise en compte d'une 
dialectique allant du détail au global dans l'intelligence du texte. Les intentions, l'ethos de l'auteur sont la condition sine qua non pour la lecture et la ré-expression de l'ironie.

Dans l'œuvre de N. Kazantzaki, on l'a vu, la reproduction de la matérialité signifiante s'avère parfois indispensable (c'est, entre autres, le cas de philipaille). En ce sens, la traduction doit être une "invention de discours". Ce qui requiert des manœuvres, des jongleries avec la lettre, les mots, les phrases, bref, la texture signifiante de l'œuvre.

Cependant, la traduction de la dérivation révèle une autre tendance, la plus fréquente et la plus applaudie. Les traducteurs se cloisonnent souvent dans l'"orthonymie", à savoir dans cette volonté de "bien écrire", de "mieux écrire", alors que Kazantzaki déplace les limites de sa langue, pour accrocher ses pensées, ironiser et inviter le lecteur à un "rôle productif". Or, si écrire n'est qu'inventer, façonner de nouveau sa langue, la traduction, comme une manière particulière d'écrire, ne doit s'envisager que comme pratique inventive et recréatrice. De la sorte, le traducteur se doit d'être prêt à "refaçonner" sa langue, toujours sur les traces de l'écrivain. Cela ne veut pas dire que nous plaidons pour une traduction-écriture "désordonnée", esthétiquement inacceptable, laide, et "malencontreuse". Au contraire, nous croyons que l'ironie doit se rendre avec bonheur et intelligence pour ne pas heurter "le génie de l'écrivain", pour ne pas attenter au tissu discursif de l'œuvre. Et cela dépend toujours de l'écrivain, de la créativité-imagination du ré-écrivain-traducteur et de la flexibilité et "des marges de manœuvre" que la langue d'arrivée offre à ce dernier.

\section{Notes}

Arsaye, Jean-Pierre (2004): Français-Créole/Créole-Français. De la traduction; éthique, pratiques, problèmes, enjeux. Paris. (= Sémantiques).

Bacry, Patrick (1992): Les figures de style et autres procédés stylistiques. Paris. (= Sujets).

Bakhtine, Mikhaïl (1978): Esthétique et théorie du roman. Traduit du russe par Daria Olivier. Paris. (= Bibliothèque des idées).

Berman, Antoine (1985/1999): La traduction et la lettre, ou, L'auberge du lointain. Paris. (= L'ordre philosophique).

Berrendonner, Alain (1982): Éléments de pragmatique linguistique. Paris. (= Propositions).

Berrendonner, Alain (2002): "Portrait de l'énonciateur en faux naïf". SEMEN. Revue de sémio-linguistique des textes et discours 15: Figures du discours et ambiguité: 113-125.

Chevalier, Jean-Claude/Delport, Marie-France (1995): L'Horlogerie de Saint-Jérôme. Problèmes linguistiques de la traduction. Paris. (= Sémantiques).

Constantinou, Maria (2006): La connotation à l'épreuve de la traduction littéraire. Une ouvre de N. Kazantzaki et "ses autres versants". Essai de sémantique textuelle. Thèse de Doctorat, Université de Franche-Comté. Besançon.

Ducrot, Oswald (1984): Le dire et le dit. Paris. (= Propositions).

Dupriez, Bernard (1984): Gradus. Les procédés littéraires (Dictionnaire). Paris.

Hamon, Philippe (1994): L'ironie littéraire. Essai sur les formes de l'écriture oblique. Paris.

Jankelevitch, Vladimir (1997): L'ironie. Paris.

Jeandillou, Jean-François (1990/1997): L'Analyse textuelle. (=Cursus).

Kerbrat-Orecchioni, Catherine (1978): "Problèmes de l'ironie". In: Kerbrat-Orecchioni, Catherine et al. (eds.)(1978): L'ironie. Lyon: 10-46. (=Linguistique et sémiologie 2).

Landheer Ronald (1998): "L'assertion ironique. Une information à rebours". In: Forsgren, Mats/Jonasson, Kerstin/Kronning, Hans (eds.)(1998): Prédication, assertion, information. Actes du colloque d'Uppsala en linguistique française, 6-9 juin 1996. Uppsala: 299-306. (= Studia Romanica Upsaliensia 56).

Mateo, Marta (1995): "The Translation of Irony". Meta, 40/1: 171-177.

Meschonnic, Henri: (1999): Poétique du traduire. Verdier. 
Perrin, Laurent (1996): L'ironie mise en trope. Du sens des énoncés hyperboliques et ironiques. Paris. (= Argumentation, sciences du langage)

Rastier, François (1987): Sémantique interprétative. Paris. (= Formes sémiotiques).

Rastier, François (1989): Sens et textualité. Paris. (= Langue, linguistique, communication).

Ricalens-Pourchot, Nicole (2003): Dictionnaire des figures de style. Paris.

Schoentjes, Pierre (1993): Recherche de l'ironie et ironie de la recherche. Gent. (= Werken uitgegeven door de Faculteit van de letteren en wijsbegeerte).

\section{Corpus}

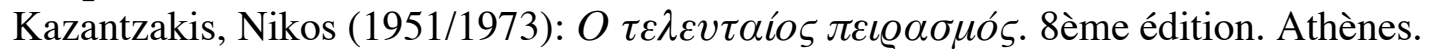

Kazantzaki, Nikos (1959/2001): La dernière tentation du Christ. Traduit du grec par Michel Saunier. Paris. (= Pocket).

Kazantzakis, Nikos (1960/1998): The last Temptation of Christ. Traduit du grec par Peter A. Bien. New York. 